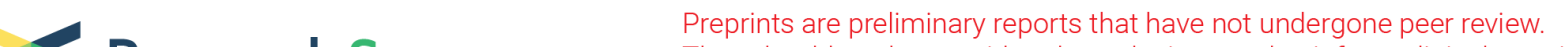 Research Square
or referenced not be considered conclusive, used to inform clinical practice,
os validated information.
}

\section{The frequency and inter-relationship of PD-L1 expression and tumour mutational burden across multiple types of advanced solid tumours in China}

\section{Yanhui Chen}

Capital Medical University Affiliated Beijing Ditan Hospital

\section{Yating Wang}

Genecast precision medicine technology institute

Hongli Luo

Genecast precision medicine technology institute

\section{Xue Meng}

Genecast precision medicine technology institute

\section{Wei Zhu}

Genecast precision medicine technology institute

\section{Di Wang}

Genecast precision medicine technology institute

\section{Henghui Zhang}

Capital Medical University Affiliated Beijing Ditan Hospital

Hui Zeng ( $\square$ zenghui@ccmu.edu.cn )

Capital Medical University Affiliated Beijing Ditan Hospital

\section{Research}

Keywords: PD-L1, TMB, PD-1, solid tumour, immunotherapy

Posted Date: April 10th, 2020

DOI: https://doi.org/10.21203/rs.3.rs-22028/v1

License: (c) (1) This work is licensed under a Creative Commons Attribution 4.0 International License. Read Full License

Version of Record: A version of this preprint was published at Experimental Hematology \& Oncology on August 3rd, 2020. See the published version at https://doi.org/10.1186/s40164-020-00173-3. 


\section{Abstract}

Background: PD-L1 expression and tumour mutational burden (TMB) have been demonstrated to be associated with the responses of multiple tumours to $\mathrm{ICl}$ therapy. Their prevalence and correlations in various types of advanced solid tumours from Chinese patients warrant further study.

Methods: PD-L1 expression, TMB, PD-1+ Tils infiltration and the relationships among them were assessed in 6,668 advanced solid tumour specimens across 25 tumour types. The relationships among PD-L1 expression, $\mathrm{TMB}, \mathrm{PD}-1^{+}$Tils and $\mathrm{CD} 8^{+} \mathrm{T}$ cell infiltration and the therapeutic effect of PD- 1 inhibitors were analysed in NSCLC samples.

Results: PD-L1 expression and TMB varied widely among tumour types. PD-L1 expression and TMB was not significantly correlated within most cancer subtypes, and they showed only a small association across all specimens (Spearman $\mathrm{R}=0.059$ ). $\mathrm{PD}-\mathrm{1}^{+}$Tils infiltration was correlated with PD-L1 expression across all samples (Spearman $\mathrm{R}=0.3056$ ). However, $\mathrm{PD}-\mathrm{1}^{+}$Tils infiltration and TMB were not correlated. In NSCLC samples, CD $8^{+}$T cell infiltration was correlated with PD-1 ${ }^{+}$Tils infiltration and PD-L1 expression but not with TMB (Spearman R $=0.4117,0.2045,0.0007$ ). Patients in the CR/PR group (anti-PD-1 therapy) had higher levels of PD-L1 expression, TMB, PD-1 ${ }^{+}$Tils and $C D 8^{+} \mathrm{T}$ cell infiltration, and more patients in this group exhibited elevated levels of multiple biomarkers.

Conclusions: Our results showed the PD-L1 expression and TMB in various types of advanced solid tumours in Chinese patients and their relationship with PD-1 ${ }^{+}$Tils and $C D 8^{+} T$ cell infiltration, which may inform ICl treatment.

\section{Background}

Tumour immunotherapy has been progressing rapidly, especially immune checkpoint inhibitor (ICI) therapy. At present, not many ICls are approved for use in China, and their indications are still few. Most ICls and indications are still in clinical trials. However, recent studies have found that some solid tumours in China or Asia have better efficacy after ICI treatment than in other regions, such as oesophageal cancer, heptocellular carcinoma, gastric or gastroesophageal junction (G/GEJ) adenocarcinoma [1-3]. The possible reasons have not been fully revealed.

Previous studies have identified a number of distinct biomarkers to predict the efficacy of ICI treatment, of which the expression of PD-L1 on tumour cells or tumour-infiltrating immune cells and the tumour mutational burden have become the most widely used biomarkers [4-22]. To predict the efficiency of ICls, PD-L1 and TMB have consistently been shown to represent independent and not correlated predictive variables $[17,19,23-25]$. There is dramatic variation in the frequency of PD-L1 expression and TMB between individual tumours and between different tumour types [26-30]. Therefore, analysis of the PDL1 expression and TMB between different samples or tumour types from different patient populations may be helpful for more accurate selection of responders to ICI treatment. 
PD-L1 and TMB in the tumour microenvironment, alone or in combination, can define groups of tumours that are "hypermutated and inflamed", "hypermutated", "inflamed" and "non-hypermutated and noninflamed" which might respond differently to ICls [30, 31]. CD8 or PD-1 expression is also a sign of the inflammatory response in the tumour microenvironment. A retrospective study reported that the expression of CD8 and PD-1 can also be used to predict the efficacy of ICls, and the predictions of TMB, PD-1, and CD8 together can explain the objective response rate of most tumour types after receiving ICls [32]. PD-L1, TMB, PD-1, and CD8 are each a potentially relevant link in the antitumour immune response process; however, their correlation has not been reported for large samples of multiple cancer types. Importantly, the frequency and correlation of these biomarkers in different types of solid tumours from Chinese patients have not been reported in large sample sizes.

In this study, we sought to determine the frequency and correlation of PD-L1 expression and TMB, and their correlation with Tils infiltration in tumour tissue samples across multiple tumour types from 6,668 Chinese advanced tumour patients. We also analysed the distribution of these biomarkers and their relationship with efficacy in small-sample immunotherapy cohorts.

\section{Methods}

\section{Patient recruitment and sample collection}

This study included 6,668 patients with advanced solid tumours, representing a total of 25 tumour types, who were tested for pharmacodynamics-related biomarkers at the Genecast Precision Medicine Technology Institute between March 2016 and February 2019. Each patient had a sufficient amount of tumour tissue sample for the detection of PD-L1, TMB, and PD-1. The clinical information of the patients is shown in Additional file 2: Supplementary Table 1. Samples from 347 NSCLC patients were used to study the relationship between $C D 8$ and PD-1, and 33 of them received anti-PD-1 therapy. The relationship of PD-L1, TMB, PD-1, CD8 and the efficacy of anti-PD-1 therapy was evaluated in these 33 cases. This study was conducted with the approval of the ethics committees of the Beijing Ditan Hospital affiliated with the Capital Medical University and the Genecast Precision Medicine Technology Institute. Each patient signed a written informed consent form.

\section{Immunohistochemical staining for PD-L1, PD-1, MLH1, MSH2, MSH6, and PMS2}

Paraffin-embedded tumour tissue samples were sectioned at a thickness of $4 \mu \mathrm{m}$ and transferred to coated glass slides. For PD-L1 staining, the slides were stained with a Ventana GX automated system (Ventana, AZ, USA). Antigen retrieval was performed in cell conditioning 1 . The primary antibody specific for PD-L1 (clone SP142) was diluted 1:25 and incubated for $32 \mathrm{~min}$ at room temperature to stain tumour cells. The antibody was detected with the Ventana Amplification Kit and Ventana ultraView Universal DAB Detection Kit. Digital images were captured using an Aperio Scanscope AT Turbo slide scanner under 20x 
magnification. Scoring of PD-L1 expression was performed using digital image analysis software, namely, Aperio Membrane v9 and Aperio Genie Classifier. PD-L1 expression was reported as a continuous variable with the percentage of tumour cells staining with any intensity. PD-L1 expression for each sample was also classified as negative, low-positive, or high-positive PD-L1 expression. Negative expression was defined as $<1 \%$ of tumour cells staining. Low-positive expression was $\geq 1 \%$ and $<50 \%$ of tumour cells staining. High-positive expression was $\geq 50 \%$ of tumour cells staining. These scoring systems were based on previous studies using an SP142 assay [33-35].

For PD-1, MLH1, MSH2, MSH6, and PMS2 staining, high-pressure reparation was used for antigen retrieval. Endogenous peroxidase activity was blocked by $3 \%$ hydrogen peroxide for 10 min. After repeated washes in PBS, the slides were incubated with antibodies against PD-1 (clone CAL20, ab237728, Abcam; dilution 1:250), MLH1 (clone ES05, RTU, Dako), MSH2 (Clone FE11, RTU, Dako), MSH6 (Clone EP49, RTU, Dako), and PMS2 (Clone EP51, RTU, Dako) according to the manufacturer's instructions. The binding of the primary antibodies was visualized by a mouse/rabbit hypersensitive polymer method detection system (PV-8000/6000, Zsbio, Beijing, China) at $37^{\circ} \mathrm{C}$ for $30 \mathrm{~min}$. Then, DAB was used for colour development for 6 min. Finally, the tissue sections were counterstained with haematoxylin, dehydrated, and mounted. The immune cell score for PD-1 was reported as a continuous variable with the percentage of the tumour area with any intensity of PD-1 staining. The criterion for dMMR was one or more of the four proteins MLH1, MSH2, MSH6, and PMS2 not expressed at all in tumour cells in a section. Two pathologists confirmed the quality and results of the experiment.

\section{Multiplex Immunohistochemical Staining For Pd-1 And Cd8}

\section{Paraffin-embedded tissue blocks were serially sectioned} into $3 \mu \mathrm{m}$ sections for the following procedures. The slides were deparaffinized in xylene, rehydrated, and washed in tap water before boiling in Tris-EDTA buffer (pH 9; 643901; Klinipath) for epitope retrieval/microwave treatment (MWT). Endogenous peroxidase and protein blocking were performed using Antibody Diluent/Block (72424205, PerkinElmer) for $10 \mathrm{~min}$ at room temperature. The antigens were labelled sequentially. Each round of antigen labelling consisted of three steps: primary antibody incubation, secondary antibody incubation, and TSA visualization. The antigen-labelled primary/secondary antibody and TSA 
complex were removed by MWT with Tris-EDTA buffer ( $\mathrm{pH}$ 9) at the end of each round, and then the next antigen was labelled. The slides were finished with MWT, counterstained with DAPI for 5 min, and mounted in Antifade Mounting Medium (10052; NobleRyder). The primary antibodies used were anti-PD-1 antibody (clone CAL20, ab237728, Abcam; dilution 1:100) and anti-CD8 antibody (clone 144B, ab17147, Abcam; dilution 1:25). The primary antibodies were incubated according to the manufacturer's instructions. Next, incubation with Polymer HRP Rb (PV6001 , Zsbio) or Polymer HRP Ms (PV-6002, Zsbio) was performed at $37^{\circ} \mathrm{C}$ for $10 \mathrm{~min}$. TSA visualization was performed with the Opal 7-Color IHC Kit (NEL797B001KT, PerkinElmer) containing the fluorophores DAPI, Opal 690 (PD-1) and Opal 540 (CD8) and the TSA Coumarin System (NEL703001KT; PerkinElmer). The slides were scanned using the PerkinElmer Vectra (Vectra 3.0.5, PerkinElmer). Multispectral images were unmixed using spectral libraries built from images of single-stained tissue samples for each reagent using inForm Advanced Image Analysis software (inForm 2.3.0, PerkinElmer). A selection of 15 representative original multispectral images was used to train the inForm software (tissue segmentation, cell segmentation, phenotyping tool, and positivity score). All of the settings applied to the training images were saved in an algorithm to allow batch analysis of multiple original multispectral images of the same tissue. Two pathologists confirmed the quality and results of the experiment.

Dna Extraction And Targeted Gene Capture Sequencing 
DNA extraction from the FFPE tumour specimens and targeted gene capture sequencing for the TMB test were performed using the standard protocols mentioned previously [36]. A total of $2 \mathrm{ml}$ of whole blood was collected from each patient, and DNA from the peripheral blood lymphocytes was extracted as a normal control. DNA libraries were captured with a designed Genescope panel of 543 genes (Genecast, Beijing, China) that included major tumour-related genes, covering $1.7 \mathrm{Mb}$ of the genomic region. The captured samples were subjected to Illumina NovaSeq 6000 platform using the pair-end sequencing method.

\section{Bioinformatics Pipeline And Tumour Mutation Burden Analysis}

The bioinformatics pipeline and tumour mutation burden analyses were performed using the standard protocols mentioned previously [36]. All base substitutions, short insertions, and deletions were initially recorded before filtering. The generated candidate mutations were annotated using Annovar software tools [37] and subsequently filtered using genomic databases such as Catalogue of Somatic Mutations in Cancer (COSMIC), the Short Genetic Variations database (dbSNP), and the Exome Aggregation Consortium (ExAC).

The TMB was defined as the number of somatic, coding, base substitutions, and indel mutations per megabase of the genome examined. All base substitutions and indels in the coding region of the targeted genes, including synonymous alterations, were initially counted before filtering as described above. Alterations that were predicted to be germline by the somatic - germline zygosity algorithm were not counted. Known germline alterations in dbSNP were not counted. Germline alterations occurring with two or more counts in the ExAC database were not counted [38]. To calculate the TMB per megabase, the total number of mutations counted was divided by the size of the coding region of the targeted territory. TMB was reported as a continuous variable. According to the TMB level, the patients were divided into three groups: high, moderate, and low. The grouping criteria were based on the 75th percentile and 25th percentile of this batch of data. Then, a TMB level that was greater than or equal to the 75th percentile was defined as high. A TMB level less than the 25th percentile was defined as low, and the moderate level occurred between the 25 th and 75 th percentiles.

\section{Validation Of Tmb Test By Whole-exome Sequencing (wes)}

WES was performed for tumour samples and matched normal control samples from 526 patients on the Illumina NovaSeq 6000 platform using the pair-end sequencing method. High-quality paired-end reads were aligned to the hg19 reference genome using the Burrows-Wheeler Aligner (BWA). The VarDict and FreeBayes programs were used for single nucleotide variation (SNV) and indel calling while the ANNOVAR assay was used for the functional annotation of genetic variants. The somatic SNVs and indels were filtered as previously reported [28]. For the determination of TMB, the number of somatic nonsynonymous 
SNVs in the whole exome (with depth $>40 \mathrm{X}$ and allele frequency $\geq 0.05$ ) detected by next generation sequencing (NGS) was quantified. Alterations known to be oncogenic drivers were excluded. TMB was measured in mutations per Mb. The validation result show that the TMB value based on targeted gene capture sequencing was consistent with the WES-based TMB value (Additional file 1: Supplementary Fig. 1).

\section{Statistical Analyses}

Statistical analyses were conducted using GraphPad Prism (version 8.2.0, La Jolla, CA, USA) and SPSS version 22.0 (SPSS, Inc., Chicago, IL, USA). Correlations between two markers were tested using the Spearman or Pearson correlation tests. The nonparametric Mann-Whitney $U$ test was used to test the significance of differences between two populations. The Kruskal-Wallis test was used to test the significance of differences among three populations. All reported $P$-values are two-tailed, and for all analyses, $P \leq 0.05$ was considered significant unless otherwise specified. A heat map and cluster analysis were implemented using R software (v3.5.1) and ComplexHeatmap (bioconductor) package.

\section{Results}

\section{PD-L1 expression and TMB landscape across tumour types}

In total, 6,668 patients with advanced tumours representing 25 tumour types were enrolled in the study, and samples with paired PD-L1 expression and TMB value were obtained during the course of standard clinical care. Summary PD-L1 qualitative IHC data are shown in Fig. 1A. PD-L1 expression varied widely among the tumour types examined. Nasopharyngeal and thymic carcinoma had the highest frequency of PD-L1 positivity $(75 \%, 68 \%)$, whereas small bowel carcinoma had the lowest frequency of PD-L1 positivity (9\%). Across all samples, $3.6 \%$ of specimens were PD-L1 high-positive (defined as $\geq 50 \%$ tumour cells staining positive for PD-L1). Among the distinct tumour types, nasopharyngeal and thymic carcinomas had the highest frequency of PD-L1 high-positive samples $(51 \%, 46 \%)$, whereas endometrial cancer had the lowest (0\%) (Additional file 1: Supplementary Fig. 2).

The median TMB across all samples was 5.08 mutations/Mb (IQR 1.99-8.89). Summary TMB data for different tumour types are shown in Fig. 1B. The median TMB for each tumour type ranged from 1.27 mutations/Mb in GIST cancer to 34.36 in colorectal (dMMR) cancer. dMMR was detected in 23 tumour types, and tumours with dMMR had the highest TMBs $(P<0.0001)$ (Additional file 1: Supplementary Fig. 3A-B). Small cell lung cancer had the highest TMBs of the non-dMMR cancers. However, PD-L1 expression did not differ between dMMR tumours and pMMR tumours $(P=0.779)$ (Additional file 1: Supplementary Fig. 3C). Cancers associated with mutagens (NSCLC-Squamous, urothelial cancer) generally had the higher TMBs of any tumour type.

\section{Relationship of PD-L1 expression and TMB}


Figure 2A shows TMB for all PD-L1-negative, PD-L1-low-positive, and PD-L1-high-positive specimens. PDL1-high-positive specimens had higher TMBs than PD-L1-low-positive and negative specimens (both $P<$ 0.0001). Across all individual specimens examined, there was a small but positive association between the PD-L1 expression and TMB (Spearman $\mathrm{R}=0.059, P<0.0001$ ). However, the relationship between these 2 biomarkers was not consistent across tumour types (Additional file 2: Supplemental Table 2). The strongest association between PD-L1 expression and TMB was within endometrial and neuroendocrine cancers (Spearman R all >0.3). There was also a weak but positive association between PD-L1 expression and TMB among cervical, gastric (pMMR), HNSCC, NSCLC (non-squamous), NSCLC (squamous) and sarcomas (Spearman R all < 0.3). However, PD-L1 expression and TMB did not correlate among most other tumour types.

Figure 2B shows proportion of samples based on PD-L1-high and TMB-high classifications for different tumour types. Nasopharyngeal cancer had the highest proportion of samples with both PD-L1-highpositive specimens and high TMB (14\%). However, endometrial, neuroendocrine, ovarian, gallbladder, breast, urothelial, gastric (dMMR), colorectal (dMMR), and colorectal (pMMR) cancers had no samples with both PD-L1-high-positive specimens and high TMB.

\section{PD-1 + Tils infiltration is related to PD-L1 expression but not to TMB}

Figure $3 \mathrm{~A}$ shows the differences in PD-1+ Tils infiltration between all PD-L1-negative/low-positive and PDL1-high-positive specimens, and Fig. 3B shows that between all TMB-moderate/low and TMB-high specimens. PD-L1-high-positive specimens had higher PD-1+ Tils infiltration than PD-L1-low-positive and negative specimens $(P<0.0001)$. However, there was no difference in PD- $1^{+}$Tils infiltration between TMBmoderate/low and TMB-high specimens $(P=0.9991)$. Across all individual samples, there was a positive association between the PD- $1^{+}$Tils infiltration and the PD-L1 expression (Spearman R $=0.3056, P<$ 0.0001) (Additional file 2: Supplemental Table 3). However, the relationship between these two biomarkers was not consistent across tumour types. The PD-1 ${ }^{+}$Tils infiltration and PD-L1 expression did not correlate within cervical, colorectal (dMMR), endometrial, gastric (dMMR), GIST, glioblastoma, small bowel, thymic, and urothelial cancers. There was a positive association between PD-1 ${ }^{+}$Tils infiltration and PD-L1 within other tumour types, and the strongest association was within breast, gallbladder, HNSCC, melanoma, and neuroendocrine cancers (Spearman R all >0.4). There was no correlation between the PD- $1^{+}$Tils infiltration and TMB, whether across all individual samples or across tumour types (Additional file 2: Supplemental Table 4).

\section{CD8 + T cell infiltration is related to PD-1+ Tils infiltration and PD-L1 expression but not to TMB}


We labelled CD8 and PD-1 proteins on the same slides from 347 NSCLC samples by multiplex immunohistochemistry and analysed the content of $\mathrm{CD} 8^{+} \mathrm{T}$ cells, PD- $1^{+} \mathrm{Tils}$, and $\mathrm{CD} 8^{+} \mathrm{PD}-1^{+} \mathrm{T}$ cells (Fig. 4A). The frequency of PD- $1^{+}$Tils, $C D 8^{+} T$ cells, and $C D 8^{+} P D-1^{+} T$ cells across all individual samples varied, and their median frequency was 5.5\% (IQR 2.9\%-8.9\%), 1.7\% (IQR 0.6\%-4.2\%), and 0.1\% (IQR $0 \%-0.5 \%$ ) respectively. There was a positive association between $\mathrm{CD} 8^{+} \mathrm{T}$ cells and PD- $1^{+}$Tils (Spearman $\mathrm{R}=0.4117, P<0.0001$ ) (Fig. 4B). However, the median proportion of CD8 ${ }^{+} \mathrm{PD}-1^{+} \mathrm{T}$ cells in total PD-1 ${ }^{+}$Tils and total CD8 ${ }^{+} \mathrm{T}$ cells was not high, 7.8\% (IQR 3.6\%-14.7\%) and 2.4\% (IQR 0.5\%-7.6\%), respectively (Fig. 4C-D). Further analysis also found a positive association between CD8 ${ }^{+} \mathrm{T}$ cells and PD-L1 expression (Spearman $\mathrm{R}=0.2045, P=0.0007$ ), but there was no correlation between $\mathrm{CD}^{+} \mathrm{T}$ cells and TMB (Spearman $\mathrm{R}=0.0007, P=0.9138$ ) (Fig. 4E-F).

\section{PD-L1 expression, TMB, PD-1 + Tils, and CD8+ T cell infiltration are related to the response of anti-PD-1 therapy in NSCLC}

In the 347 NSCLC patients mentioned above, 33 of them received the anti-PD-1 therapy or anti-PD-1 therapy plus chemotherapy. All patients were EGFR/KRAS wild-type patients, including 14 cases of lung adenocarcinoma and 20 cases of lung squamous cell carcinoma, of which 10 received PD-1 inhibitor monotherapy and 23 patients received a combination of PD-1 inhibitor plus chemotherapy. Detailed clinical information on those cases is provided in Additional file 2: Supplementary Table 5. The efficacy of treatment was evaluated as objective tumour response; 1 case achieved CR (complete response), 12 cases achieved PR (partial response), 9 cases had SD (stable disease), and 11 patients had PD (progressive disease). There was no significant difference in objective response rates $(O R R=(C R+$ $\mathrm{PR}) /(\mathrm{CR}+\mathrm{PR}+\mathrm{SD}+\mathrm{PD})$ ) between the PD-1 inhibitor monotherapy group and the PD-1 inhibitor plus chemotherapy group. The ORR of the monotherapy group was $40.0 \%$, and the ORR of the PD- 1 inhibitor plus chemotherapy group was $39.1 \%$. The heat map result for the cluster analysis of each case showed that the CR/PR group had higher levels of PD-L1 expression, TMB, PD-1 ${ }^{+}$Tils infiltration, and $\mathrm{CD} 8^{+} \mathrm{T}$ cell infiltration, and most patients in this group exhibited elevated levels of multiple biomarkers. However, in the SD/PD group, the four markers were at a lower level (Fig. 5A). Generally, the levels of the three biomarkers, including TMB, PD-1 ${ }^{+}$Tils infiltration, and $\mathrm{CD} 8^{+} \mathrm{T}$ cell infiltration, were significantly higher in the $\mathrm{CR} / \mathrm{PR}$ group than in the SD/PD group $(P=0.0017, P=0.0466$, and $P=0.0396$, respectively) (Fig. 5BD). The PD-L1 expression was also higher in the CR/PR group than in the SD/PD group, but this difference was not significant $(P=0.7364)$ (Fig. 5E).

\section{Discussion}

To the best of our knowledge, this is the largest report of PD-L1 expression by IHC and TMB by targeted gene capture sequencing across multiple tumour types from Chinese advanced cancer patients. We found that PD-L1 expression and TMB varied widely among the tumour types (Fig. 1). This result is 
similar to the previous report in general [30]. However, in some tumour types the status of PD-L1 expression and TMB differed from our findings. Our study found that the PD-L1 expression and TMB levels of melanoma were lower than those reported in the previous study. This may be due to differences in the tissue origin of the tumour. Chinese melanoma is mostly mucosal rather than the UV-related hypermutated skin type. In oesophageal cancer, the positive rate of PD-L1 was much higher than that of previous reports. Recent studies have found that oesophageal cancers in China have better efficacy after $\mathrm{ICI}$ treatment than in other regions [1]. These results suggest that differential studies of ICl-related biomarkers in different populations are needed.

The relative independence of PD-L1 expression and TMB within most tumour types suggests that each biomarker could inform the use of ICl therapy in tumours with specific tumour microenvironments. Previous reports have suggested that these biomarkers could define the immunologic state of the tumour microenvironment as "hypermutated and inflamed", "hypermutated", "inflamed", or "nonhypermutated and noninflamed" based on TMB-high and PD-L1-high classifications [30, 31]. The nonhypermutated and noninflamed type of tumour may be resistant to $\mathrm{ICl}$ monotherapy, and the hypermutated and inflamed types of tumour are the best for $\mathrm{ICl}$ monotherapy. Based on the results of this study, our study found that some tumour types can be similarly classified, such as nasopharyngeal, NSCLC, HNSCC, etc. However, this classification may not be suitable for other tumour types, such as endometrial, breast, urothelial, colorectal, etc. (Fig. 2). There were no or few hypermutated and inflamed samples in these tumour types. For these tumour types, their efficacy for immunotherapy alone requires validation by clinical trials. The immunotherapeutic intervention strategy that combines other treatments also needs to be considered, and the biomarkers associated with their efficacy may need to be evaluated from other perspectives.

PD-1 expression has also shown predictive power in evaluating the efficacy of ICl therapy [32, 39]. Our study also found that in samples of most tumour types there was a positive association between the PD$1^{+}$Tils infiltration and PD-L1 expression (Fig. 3) (Additional file 2: Supplemental Table 3); in addition, PD$1^{+}$Tils did correlate with CD8 ${ }^{+} \mathrm{T}$ cells in the NSCLC samples tested (Fig. 4). In T cells, PD-1 expression may indicate cell activation. Similar to PD-1, PD-L1 expression can also be a marker of immune activation. The expression of PD-L1 on tumours and in the tumour microenvironment is mostly dependent on the immune activation pathway of IFN-r. IFN-r produced by effector T cells soon after but not before activation of immune response is the major inducer of PD-L1 expression at the transcription level $[40,41]$. However, PD-1 is generally expressed on many types of tumour-infiltrating lymphocytes in tumour tissues and not only on effector $T$ cells. Our study found that the proportion of CD8 ${ }^{+} P D-1^{+} T$ cells in total PD-1 ${ }^{+}$Tils varied greatly in different samples, but overall, it was not high (median $7.8 \%$ (IQR 3.6\%-14.7\%)) (Fig. 4). There are other immune cells in large amounts in the microenvironment that express PD-1. If there are a large number of Tregs or MDSCs in these immune cells, the efficacy of ICI treatment may not be good. This hypothesis requires further research to provide practical evidence. In this study, we found that PD-1 ${ }^{+}$Tils infiltration did not correlate with TMB (Fig. 3) (Additional file 2: Supplemental Table 4). Interestingly, recent studies have shown that the efficacy of anti-PD-1 therapy is not significantly associated with TMB [42-44]. In these clinical trials, the TMB levels were not 
significantly associated with objective response rates, progression-free survival, and overall survival. Our results provide a reasonable explanation for the results of these clinical trials, which is that TMB-H tumours may not have enough drug-reactive PD- $1^{+}$Tils to elicit a therapeutic response of PD- 1 inhibitors during initial treatment.

A retrospective study reported that the predictions of TMB, PD-1, and CD8 together can explain the objective response rate of most tumour types after receiving ICls [32]. Our research found a similar phenomenon. In the NSCLC cases receiving anti-PD-1 therapy, we found that the samples of the CR/PR group had higher levels of PD-L1 expression, TMB, PD-1 ${ }^{+}$Tils infiltration, and CD $8^{+} \mathrm{T}$ cell infiltration (Fig. 5), and most of the individual samples that were multiple biomarkers were at a high level. Some of these samples could be clustered into "hypermutated and inflamed", or "hypermutated", or "inflamed" types as mentioned above. However, there was no such phenomenon in the SD/PD group, and most of the samples in this group could be clustered into the "nonhypermutated and noninflamed" type. We believe that the classification of "hypermutated and inflamed", "hypermutated", or "inflamed" should not be based only on the results of TMB and PD-L1 but rather should be based on the results of more biomarkers such as TMB, PD- L1, PD-1, and CD8, which may be more effective in helping to find more responders to PD-1 inhibitor therapy.

The advantages of this study include the use of clinically validated analytical methods in a CAP-certified laboratory to report PD-L1 expression, TMB, and other markers in a large number of representative clinical samples from Chinese patients. A limitation is that the sample used to observe the therapeutic

relationship between biomarkers and $\mathrm{ICl}$ was derived from only one tumour type, and the sample size was small. The role of these markers in the treatment of $\mathrm{ICl}$ in different tumour types requires further exploration and validation.

\section{Conclusions}

In summary, we analysed PD-L1 expression, TMB, and Tils infiltration and their correlations in various types of advanced solid tumours from Chinese patients. These data may inform ICI treatment and help identify the type of tumour or individual patient most likely or least likely to benefit from ICl treatment.

\section{List Of Abbreviations}

Programmed death ligand-1 (PD-L1); Programmed death-1 (PD-1); Tumour mutation burden (TMB); Immune checkpoint inhibitor (ICl); Mismatch repair deficiency (dMMR); MMR-proficient (pMMR); Next generation sequencing (NGS); Whole-exome sequencing (WES); Tumour-infiltrating lymphocytes (Tils); Complete response (CR); Partial response (PR); Stable disease (SD); Progressive disease (PD).

\section{Declarations}

\section{Ethics approval and consent to participate}


The study was conducted with the approval of the ethics committees of the Beijing Ditan Hospital affiliated to the Capital Medical University and the Genecast Precision Medicine Technology Institute. Each patient signed a written informed consent form.

\section{Consent for publication}

Not applicable.

\section{Availability of data and material}

Not applicable.

\section{Competing interests}

The authors declare that they have no competing interests.

\section{Funding}

This work was supported by the National Key Sci-Tech Special Project of China (No. 2018ZX10302207).

\section{Authors' contributions}

Yanhui Chen wrote the first draft of the manuscript; Hui Zeng and Henghui Zhang contributed to the conception and design of the research; Yanhui Chen, Yating Wang and Hongli Luo contributed to the analysis and interpretation of the data; Xue Meng, Wei Zhu, Di Wang contributed to the experiment and analysis of the data. All authors critically revised the manuscript and agreed to be fully accountable for ensuring the integrity and accuracy of the work and read and approved the final manuscript.

\section{Acknowledgements}

Not applicable.

\section{References}

1. Chen J, Luo S, Qin S, Cheng Y, Li Z, Fan Y, Yuan X, et al. Pembrolizumab vs chemotherapy in patients with advanced/metastatic adenocarcinoma (AC) or squamous cell carcinoma (SCC) of the esophagus as second-line therapy: Analysis of the Chinese subgroup in KEYNOTE-181. Annals of Oncology 2019;30:v294. 2019 ESMO.

2. Tabernero J. Pembrolizumab with or without Chemotherapy Versus Chemotherapy in Advanced G/GEJ Adenocarcinoma: The Phase 3, KEYNOTE-062 Study. Journal of Clinical Oncology 2019;37:233s. 2019 ASCO.

3. Finn RS, Ryoo BY, Merle P, Kudo M, Bouattour M, Lim HY, Breder V, et al. Pembrolizumab As SecondLine Therapy in Patients With Advanced Hepatocellular Carcinoma in KEYNOTE-240: A Randomized, Double-Blind, Phase III Trial. J Clin Oncol 2020;38:193-202. 
4. Patel SP, Kurzrock R. PD-L1 Expression as a Predictive Biomarker in Cancer Immunotherapy. Mol Cancer Ther 2015;14:847-856.

5. Rosenberg JE, Hoffman-Censits J, Powles T, van der Heijden MS, Balar AV, Necchi A, Dawson N, et al. Atezolizumab in patients with locally advanced and metastatic urothelial carcinoma who have progressed following treatment with platinum-based chemotherapy: a single-arm, multicentre, phase 2 trial. Lancet 2016;387:1909-1920.

6. Reck M, Rodriguez-Abreu D, Robinson AG, Hui R, Csoszi T, Fulop A, Gottfried M, et al. Pembrolizumab versus Chemotherapy for PD-L1-Positive Non-Small-Cell Lung Cancer. N Engl J Med 2016;375:18231833.

7. Taube JM, Klein A, Brahmer JR, Xu H, Pan X, Kim JH, Chen L, et al. Association of PD-1, PD-1 ligands, and other features of the tumor immune microenvironment with response to anti-PD-1 therapy. Clin Cancer Res 2014;20:5064-5074.

8. Fuchs CS, Doi T, Jang RW, Muro K, Satoh T, Machado M, Sun W, et al. Safety and Efficacy of Pembrolizumab Monotherapy in Patients With Previously Treated Advanced Gastric and Gastroesophageal Junction Cancer: Phase 2 Clinical KEYNOTE-059 Trial. JAMA Oncol 2018;4:e180013.

9. Garon EB, Rizvi NA, Hui R, Leighl N, Balmanoukian AS, Eder JP, Patnaik A, et al. Pembrolizumab for the treatment of non-small-cell lung cancer. N Engl J Med 2015;372:2018-2028.

10. Paz-Ares L, Luft A, Vicente D, Tafreshi A, Gumus M, Mazieres J, Hermes B, et al. Pembrolizumab plus Chemotherapy for Squamous Non-Small-Cell Lung Cancer. N Engl J Med 2018;379:2040-2051.

11. Socinski MA, Jotte RM, Cappuzzo F, Orlandi F, Stroyakovskiy D, Nogami N, Rodriguez-Abreu D, et al. Atezolizumab for First-Line Treatment of Metastatic Nonsquamous NSCLC. N Engl J Med 2018,378:2288-2301.

12. Gandhi L, Rodriguez-Abreu D, Gadgeel S, Esteban E, Felip E, De Angelis F, Domine M, et al. Pembrolizumab plus Chemotherapy in Metastatic Non-Small-Cell Lung Cancer. 2018;378:2078-2092.

13. Balar AV, Galsky MD, Rosenberg JE, Powles T, Petrylak DP, Bellmunt J, Loriot Y, et al. Atezolizumab as first-line treatment in cisplatin-ineligible patients with locally advanced and metastatic urothelial carcinoma: a single-arm, multicentre, phase 2 trial. Lancet 2017;389:67-76.

14. Bellmunt J, de Wit R, Vaughn DJ, Fradet Y, Lee JL, Fong L, Vogelzang NJ, et al. Pembrolizumab as Second-Line Therapy for Advanced Urothelial Carcinoma. N Engl J Med 2017;376:1015-1026.

15. Overman MJ, Lonardi S, Wong KYM, Lenz HJ, Gelsomino F, Aglietta M, Morse MA, et al. Durable Clinical Benefit With Nivolumab Plus Ipilimumab in DNA Mismatch Repair-Deficient/Microsatellite Instability-High Metastatic Colorectal Cancer. J Clin Oncol 2018;36:773-779.

16. Le DT, Uram JN, Wang H, Bartlett BR, Kemberling H, Eyring AD, Skora AD, et al. PD-1 Blockade in Tumors with Mismatch-Repair Deficiency. N Engl J Med 2015;372:2509-2520.

17. Hellmann MD, Ciuleanu TE, Pluzanski A, Lee JS, Otterson GA, Audigier-Valette C, Minenza E, et al. Nivolumab plus Ipilimumab in Lung Cancer with a High Tumor Mutational Burden. N Engl J Med 2018;378:2093-2104. 
18. Rizvi NA, Hellmann MD, Snyder A, Kvistborg P, Makarov V, Havel JJ, Lee W, et al. Cancer immunology. Mutational landscape determines sensitivity to PD-1 blockade in non-small cell lung cancer. Science 2015;348:124-128.

19. Hellmann MD, Nathanson T, Rizvi H, Creelan BC, Sanchez-Vega F, Ahuja A, Ni A, et al. Genomic Features of Response to Combination Immunotherapy in Patients with Advanced Non-Small-Cell Lung Cancer. Cancer Cell 2018;33:843-852 e844.

20. Hellmann MD, Callahan MK, Awad MM, Calvo E, Ascierto PA, Atmaca A, Rizvi NA, et al. Tumor Mutational Burden and Efficacy of Nivolumab Monotherapy and in Combination with Ipilimumab in Small-Cell Lung Cancer. Cancer Cell 2018;33:853-861 e854.

21. Snyder A, Makarov V, Merghoub T, Yuan J, Zaretsky JM, Desrichard A, Walsh LA, et al. Genetic basis for clinical response to CTLA-4 blockade in melanoma. N Engl J Med 2014;371:2189-2199.

22. Van Allen EM, Miao D, Schilling B, Shukla SA, Blank C, Zimmer L, Sucker A, et al. Genomic correlates of response to CTLA-4 blockade in metastatic melanoma. Science 2015;350:207-211.

23. Carbone DP, Reck M, Paz-Ares L, Creelan B, Horn L, Steins M, Felip E, et al. First-Line Nivolumab in Stage IV or Recurrent Non-Small-Cell Lung Cancer. N Engl J Med 2017;376:2415-2426.

24. Rizvi H, Sanchez-Vega F, La K, Chatila W, Jonsson P, Halpenny D, Plodkowski A, et al. Molecular Determinants of Response to Anti-Programmed Cell Death (PD)-1 and Anti-Programmed DeathLigand 1 (PD-L1) Blockade in Patients With Non-Small-Cell Lung Cancer Profiled With Targeted NextGeneration Sequencing. J Clin Oncol 2018;36:633-641.

25. Goodman AM, Kato S, Bazhenova L, Patel SP, Frampton GM, Miller V, Stephens PJ, et al. Tumor Mutational Burden as an Independent Predictor of Response to Immunotherapy in Diverse Cancers. Mol Cancer Ther 2017;16:2598-2608.

26. Lawrence MS, Stojanov P, Polak P, Kryukov GV, Cibulskis K, Sivachenko A, Carter SL, et al. Mutational heterogeneity in cancer and the search for new cancer-associated genes. Nature 2013;499:214-218.

27. Ciriello G, Miller ML, Aksoy BA, Senbabaoglu Y, Schultz N, Sander C. Emerging landscape of oncogenic signatures across human cancers. Nat Genet 2013;45:1127-1133.

28. Chalmers ZR, Connelly CF, Fabrizio D, Gay L, Ali SM, Ennis R, Schrock A, et al. Analysis of 100,000 human cancer genomes reveals the landscape of tumor mutational burden. Genome Med 2017;9:34.

29. Zehir A, Benayed R, Shah RH, Syed A, Middha S, Kim HR, Srinivasan P, et al. Mutational landscape of metastatic cancer revealed from prospective clinical sequencing of 10,000 patients. Nat Med 2017;23:703-713.

30. Yarchoan M, Albacker LA, Hopkins AC, Montesion M, Murugesan K, Vithayathil TT, Zaidi N, et al. PDL1 expression and tumor mutational burden are independent biomarkers in most cancers. JCI Insight 2019;4.

31. Chan TA, Yarchoan M, Jaffee E, Swanton C, Quezada SA, Stenzinger A, Peters S. Development of tumor mutation burden as an immunotherapy biomarker: utility for the oncology clinic. Ann Oncol 2019;30:44-56. 
32. Lee JS, Ruppin E. Multiomics Prediction of Response Rates to Therapies to Inhibit Programmed Cell Death 1 and Programmed Cell Death 1 Ligand 1. JAMA Oncol 2019.

33. Rittmeyer A, Barlesi F, Waterkamp D, Park K, Ciardiello F, von Pawel J, Gadgeel SM, et al. Atezolizumab versus docetaxel in patients with previously treated non-small-cell lung cancer (OAK): a phase 3, open-label, multicentre randomised controlled trial. Lancet 2017;389:255-265.

34. Fehrenbacher L, Spira A, Ballinger M, Kowanetz M, Vansteenkiste J, Mazieres J, Park K, et al. Atezolizumab versus docetaxel for patients with previously treated non-small-cell lung cancer (POPLAR): a multicentre, open-label, phase 2 randomised controlled trial. Lancet 2016;387:18371846.

35. Herbst RS, Soria JC, Kowanetz M, Fine GD, Hamid O, Gordon MS, Sosman JA, et al. Predictive correlates of response to the anti-PD-L1 antibody MPDL3280A in cancer patients. Nature 2014;515:563-567.

36. Chen Y, Liu Q, Chen Z, Wang Y, Yang W, Hu Y, Han W, et al. PD-L1 expression and tumor mutational burden status for prediction of response to chemotherapy and targeted therapy in non-small cell lung cancer. J Exp Clin Cancer Res 2019;38:193.

37. Wang K, Li M, Hakonarson H. ANNOVAR: functional annotation of genetic variants from highthroughput sequencing data. Nucleic Acids Res 2010;38:e164.

38. Lek M, Karczewski KJ, Minikel EV, Samocha KE, Banks E, Fennell T, O'Donnell-Luria AH, et al. Analysis of protein-coding genetic variation in 60,706 humans. Nature 2016;536:285-291.

39. Pare L, Pascual T, Segui E, Teixido C, Gonzalez-Cao M, Galvan P, Rodriguez A, et al. Association between PD1 mRNA and response to anti-PD1 monotherapy across multiple cancer types. Ann Oncol 2018;29:2121-2128.

40. Isogawa M, Furuichi Y, Chisari FV. Oscillating CD8(+) T cell effector functions after antigen recognition in the liver. Immunity 2005;23:53-63.

41. Brown JA, Dorfman DM, Ma FR, Sullivan EL, Munoz O, Wood CR, Greenfield EA, et al. Blockade of programmed death- 1 ligands on dendritic cells enhances $T$ cell activation and cytokine production. $J$ Immunol 2003;170:1257-1266.

42. Garassino M, Rodriguez-Abreu D, Gadgeel S, Esteban E, Felip E, Speranza G, Reck M. Evaluation of TMB In KEYNOTE-189: Pembrolizumab plus Chemotherapy vs Placebo plus Chemotherapy for Nonsquamous NSCLC. IASLC 2019 World Conference on Lung Cancer Abstract Book 2019:225.

43. C. Langer C, S. Gadgeel S, H. Borghaei H, A. Patnaik A, S. Powell S, R. Gentzler R, Yang J. KEYNOTE021: TMB and Outcomes for Carboplatin and Pemetrexed with or without Pembrolizumab for Nonsquamous NSCLC. IASLC 2019 World Conference on Lung Cancer Abstract Book 2019:225.

44. Peters S, Ramalingam S, Paz-Ares L, Bernabe Caro R, Zurawski B, Kim S, Alexandru A, et al. Nivolumab (NIVO) 1 low-dose ipilimumab (IPI) vs platinum- doublet chemotherapy (chemo) as firstline (1L) treatment (tx) for advanced non-small cell lung cancer (NSCLC): CheckMate 227 part 1 final analysis. Annals of Oncology 2019;30:v914. 2019 ESMO. 


\section{Additional File Legends}

Additional file 1: Supplementary figure 1-3 and figure legends.

Additional file 2: Supplementary table 1-5.

\section{Figures}

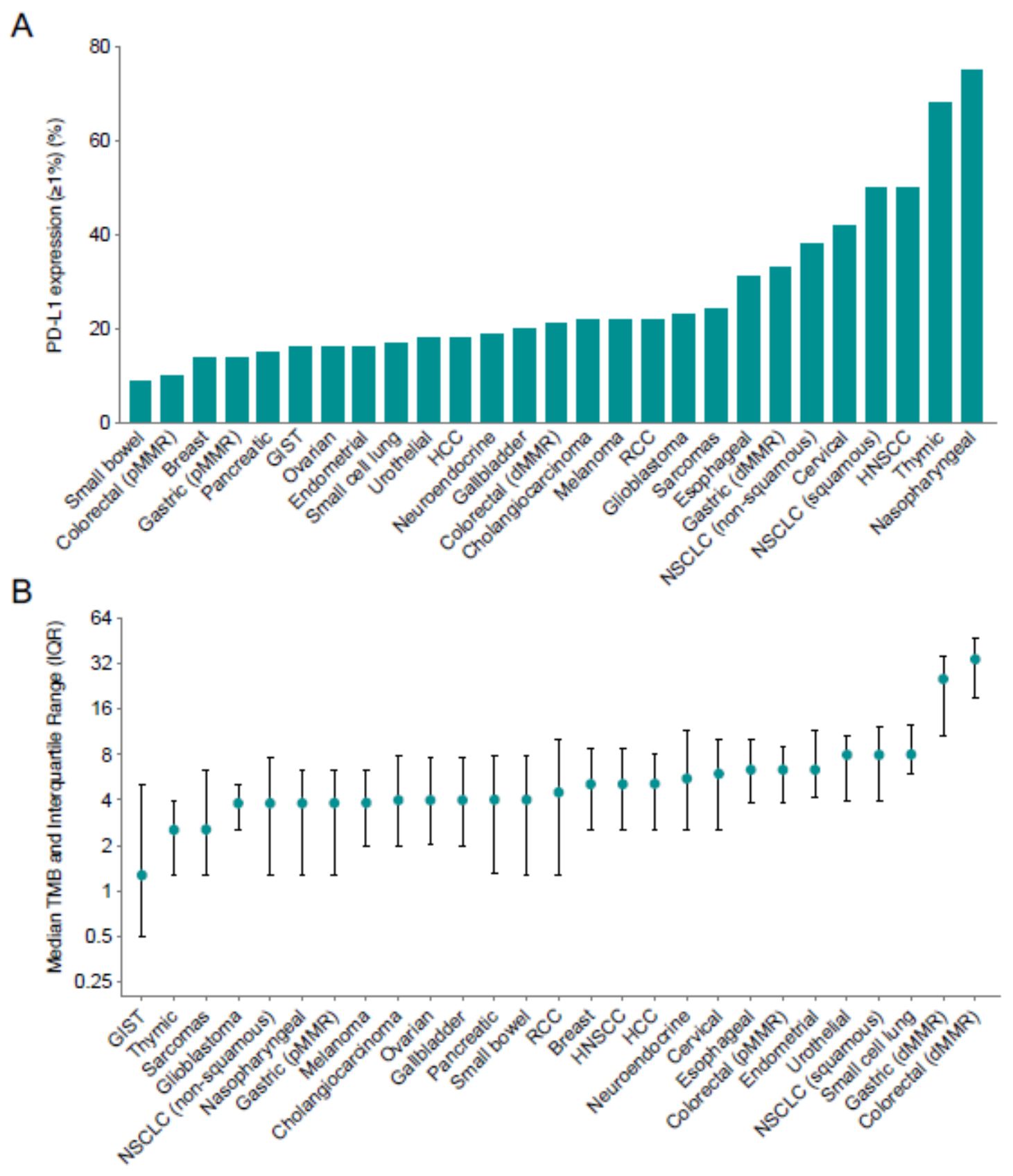

Figure 1

Landscape of PD-L1 expression and TMB across tumour types. A. Percentage of tumours with positive PD-L1 expression ( $\geq 1 \%$ ) by IHC within 25 major tumour types, from the lowest frequency of positivity 
(left) to the highest frequency (right). B. Tumours are ordered from the lowest median TMB (left) to the highest median TMB (right). dMMR, mismatch repair deficient.

A

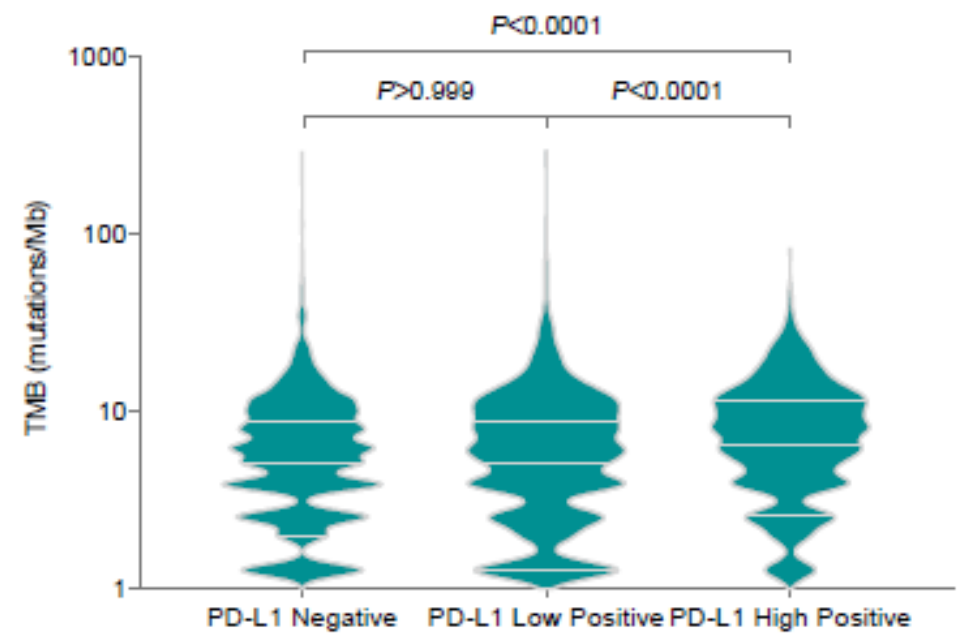

\section{B}

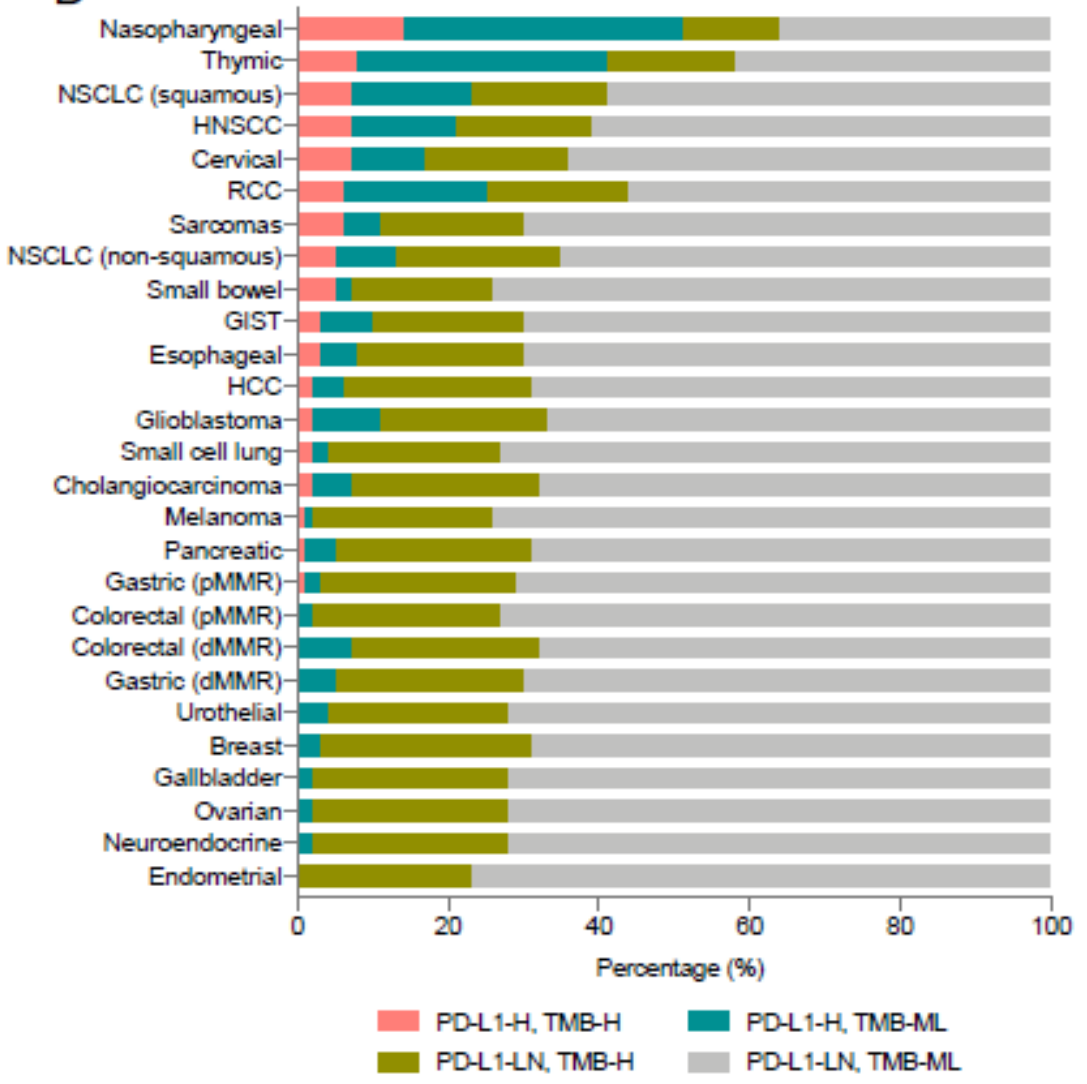

\section{Figure 3}

Relationship between TMB and PD-L1 expression. A. Differences in TMB between PD-L1-negative, lowpositive and high-positive tumours. Kruskal-Wallis test. B. Proportion of samples based on PD-L1-high and TMB-high classifications for different tumour types. PD-L1-H, PD-L1 high-positive. PD-L1-LN, PD-L1 low-positive and negative. TMB-H, TMB high. TMB-ML, TMB moderate and low. 

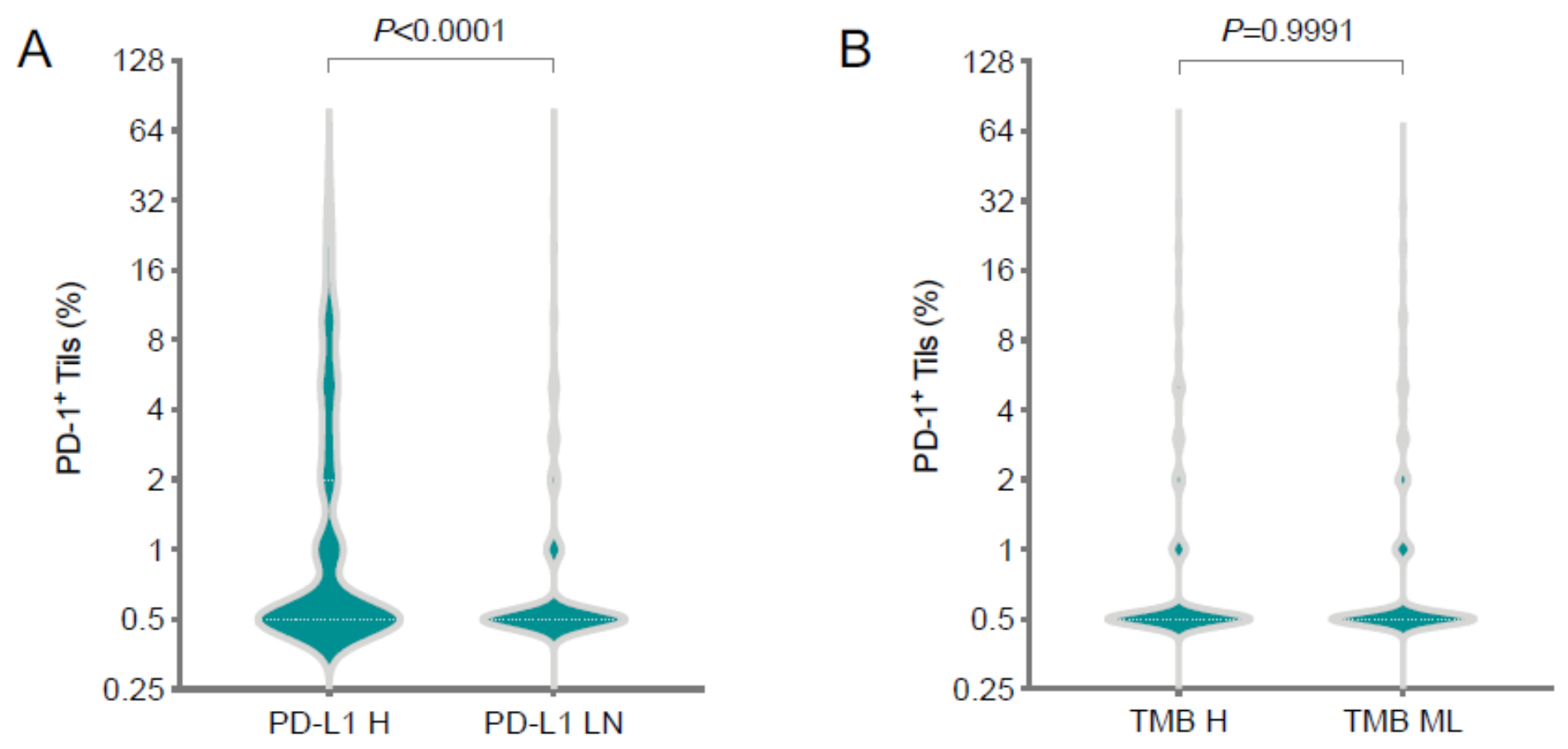

Figure 5

Differences in PD-1+ Tils infiltration between groups divided by PD-L1 expression and TMB. A. Differences in PD-1+ Tils infiltration between PD-L1-high-positive tumours and PD-L1-negative or lowpositive tumours. Mann-Whitney U test. B. Differences in PD-1+ Tils infiltration between TMB-high tumours and TMB-moderate or low tumours. Mann-Whitney $\mathrm{U}$ test. 
A

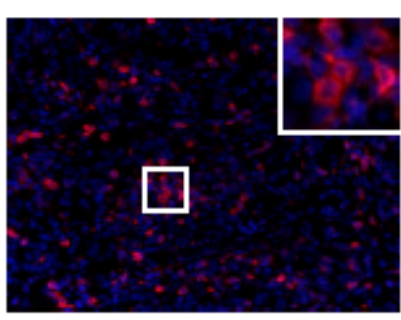

PD-1

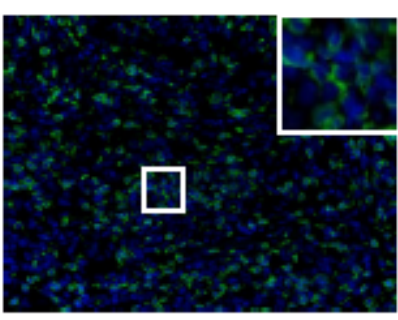

CD8

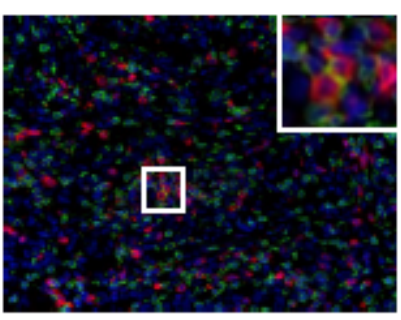

Merged
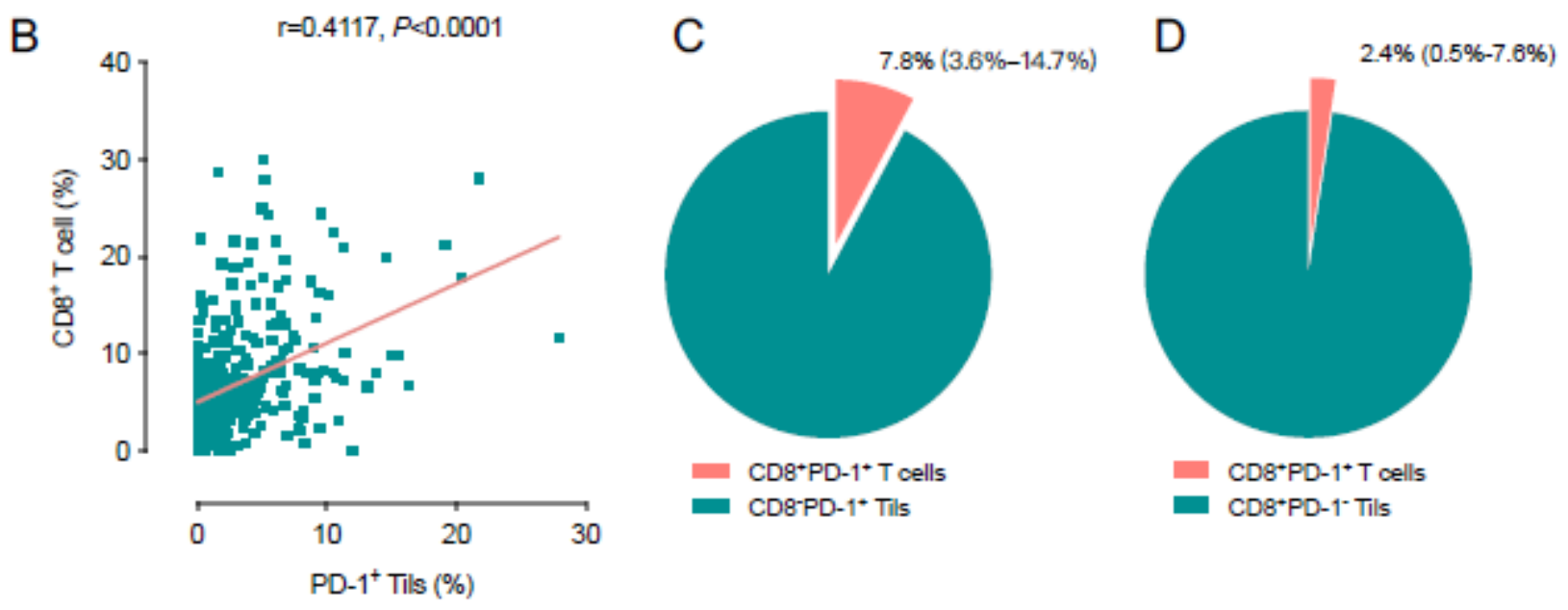

$E$

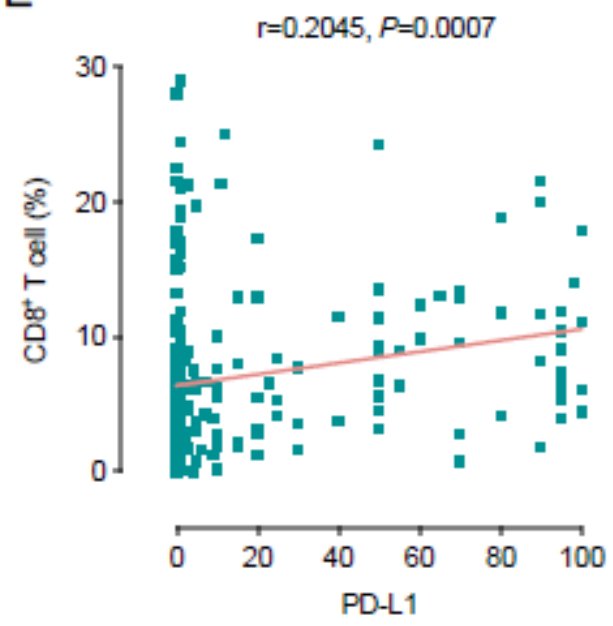

$\mathrm{F}$

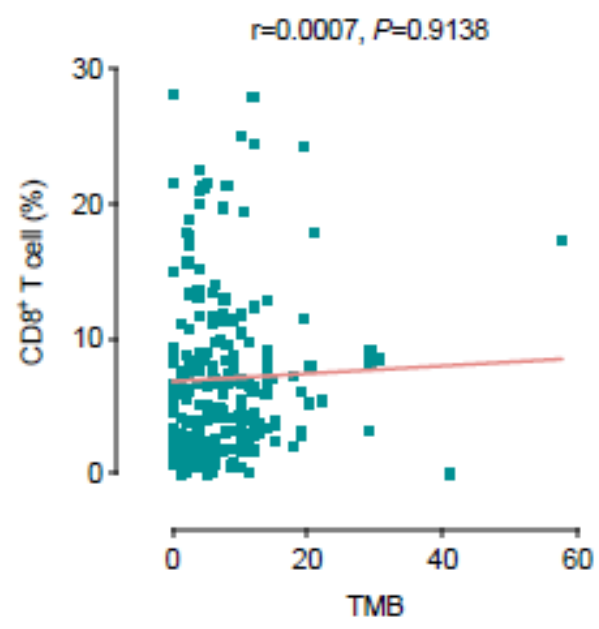

\section{Figure 7}

Relationships among CD8+ T cell infiltration, PD-1+ Tils infiltration, PD-L1, and TMB. A. Representative images of PD-1+ Tils, CD8+ T cells, and CD8+PD-1+ T cells in the same slide from a lung adenocarcinoma sample evaluated by multiplex immunohistochemical staining. 20x magnification. $B$. Correlation analysis between PD-1+ Tils and CD8+ T cells. Spearman correlation test. C-D. The median proportion of CD8+PD-1+ T cells in total PD-1+ Tils and total CD8+ T cells. In the brackets is the interquartile range (IQR). E. Correlation analysis between PD-L1 and CD8+ T cells. Spearman correlation test. F. Correlation analysis between TMB and CD8+ T cells. Spearman correlation test. 


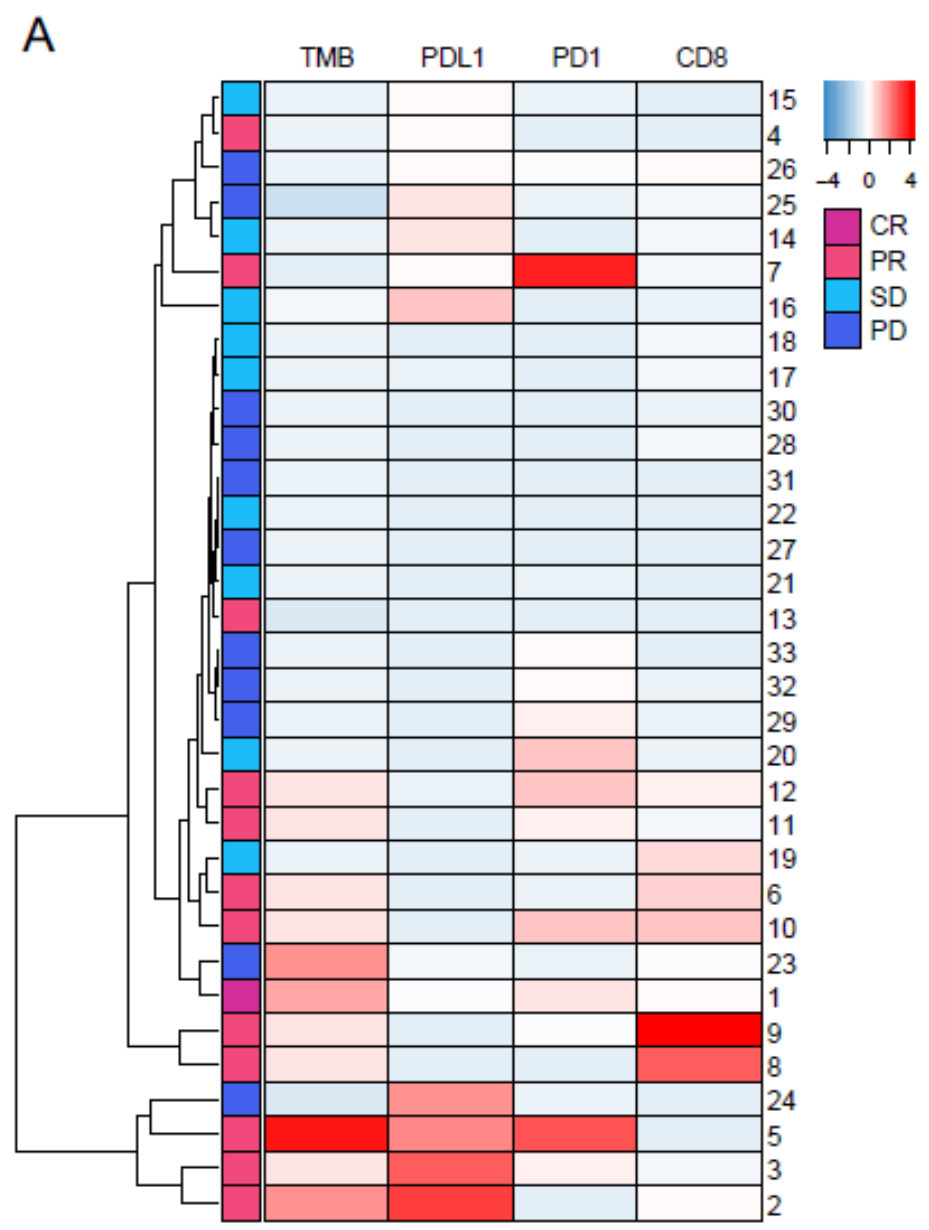

B

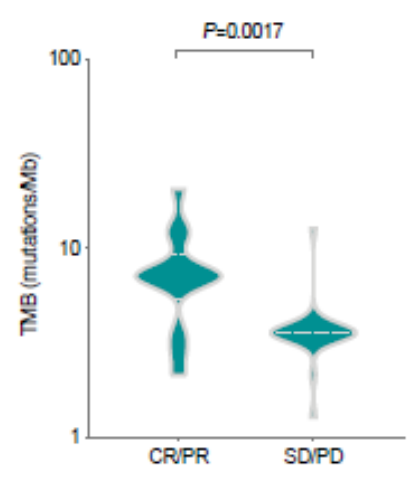

D

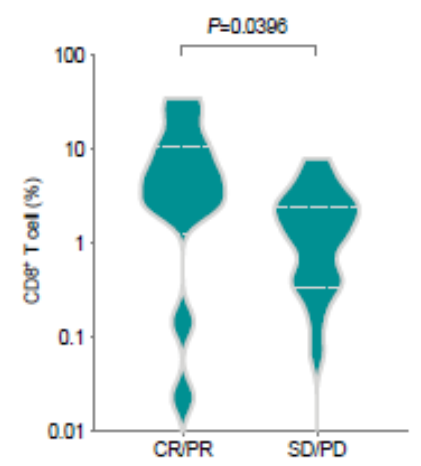

C

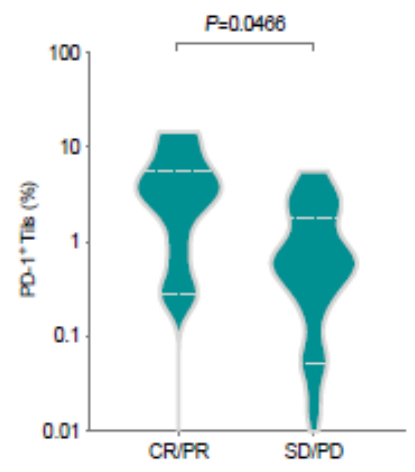

E

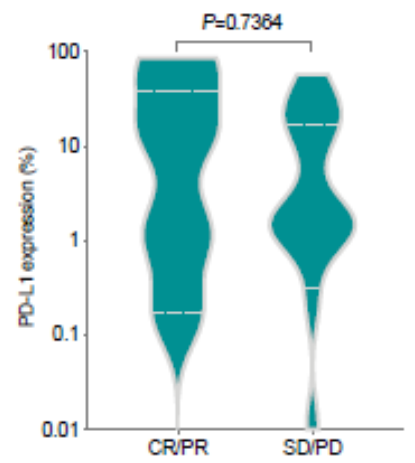

Figure 9

Relationships among PD-L1 expression, TMB, PD-1+ Tils, CD8+ T cell, and response to anti-PD-1 therapy. A. Heatmap result of the PD-L1 expression, TMB, PD-1+ Tils infiltration, and CD8+ T cell infiltration in different groups according to the response to anti-PD-1 therapy. B-E. Differences in PD-L1 expression, TMB, PD-1+ Tils infiltration, and CD8+ T cell infiltration between the CR/PR group and SD/PD group. Mann-Whitney U test. CR, complete response. PR, partial response. SD, stable disease. PD, progressive disease.

\section{Supplementary Files}

This is a list of supplementary files associated with this preprint. Click to download.

- Additionalfile1.pdf

- Additionalfile2.pdf

- Additionalfile2.pdf

- Additionalfile1.pdf 